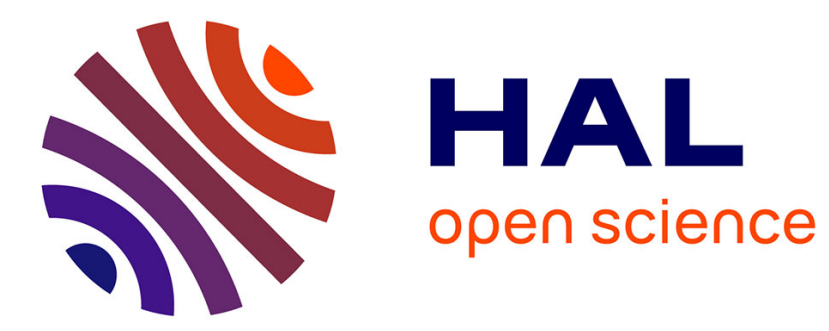

\title{
La création des UHSA : une nouvelle régulation de l'enfermement?
}

Benoît Eyraud, Livia Velpry, Claude-Olivier Doron, Camille Lancelevée, Litzler Alexandre, Protais Caroline, Sébastien Saetta

\section{- To cite this version:}

Benoît Eyraud, Livia Velpry, Claude-Olivier Doron, Camille Lancelevée, Litzler Alexandre, et al.. La création des UHSA : une nouvelle régulation de l'enfermement?. Déviance et Société, 2015, 39 (4), pp.429-453. 10.3917/ds.394.0429 . halshs-01293271

\section{HAL Id: halshs-01293271 \\ https://shs.hal.science/halshs-01293271}

Submitted on 29 Mar 2016

HAL is a multi-disciplinary open access archive for the deposit and dissemination of scientific research documents, whether they are published or not. The documents may come from teaching and research institutions in France or abroad, or from public or private research centers.
L'archive ouverte pluridisciplinaire HAL, est destinée au dépôt et à la diffusion de documents scientifiques de niveau recherche, publiés ou non, émanant des établissements d'enseignement et de recherche français ou étrangers, des laboratoires publics ou privés. 


\title{
La création des UHSA : une nouvelle régulation de l'enfermement ?
}

\author{
Collectif Contrast
}

Benoît Eyraud (Université Lyon 2 - CMW), Livia Velpry (Université Paris 8 - Cermes 3), Claude-Olivier Doron (Université ParisDiderot - Sphere), Camille Lancelevée (EHESS Iris), Alexandre Litzler (Université Paris-Ouest Nanterre), Caroline Protais (Cermes3), Sébastien Saetta (Cermes 3)

\section{livia.velpry@univ-paris8.fr et benoit.eyraud@ish-lyon.cnrs.fr}

\section{PRE-PRINT AVANT EPREUVES DE L'ARTICLE PARU DANS DEVIANCE ET} SOCIETE, ${ }^{\circ}{ }^{4}$, vol. 39, 2015

En 2010, à l'ouverture de la première UHSA, destinée à hospitaliser en psychiatrie des personnes détenues, critiques sécuritaires et défenseurs d'un outil d'égal accès aux soins s'opposent. Notre analyse interdisciplinaire de ce développement controversé croise la force normative des règles autorisant l'enfermement, le contexte de leur élaboration et leurs usages sociaux. À partir de textes juridiques et administratifs et d'entretiens avec les acteurs, nous montrons que la régulation dichotomique issue du $\mathrm{XIX}^{\mathrm{e}}$ siècle et l'essor des services psychiatriques en prison éclairent comment les règles élaborées pour les UHSA participent aux recompositions contemporaines du sens de l'enfermement.

\section{Introduction}

En mai 2010 ouvre la première Unité Hospitalière Spécialement Aménagée (UHSA), à l'hôpital du Vinatier, à Lyon-Bron. Huit autres unités s'y ajoutent dans les quatre années suivantes ${ }^{1}$. Ces unités psychiatriques sécurisées d'un type nouveau sont destinées à hospitaliser les détenus pour des soins en santé mentale. Implantées à l'hôpital mais entourées d'un mur d'enceinte pénitentiaire, ces unités empruntent à deux institutions, la prison et l'hôpital psychiatrique, qui se sont construites historiquement en opposition. Elles semblent ainsi produire un assemblage original, hybride, de ces deux institutions.

C'est d'ailleurs une telle hybridation que dénoncent les détracteurs de ces unités, surnommées dans la presse du début des années 2000 « hôpital-prison ». Acteurs de la société civile et professionnels de la psychiatrie, mais également psychiatres responsables de ce type

\footnotetext{
${ }^{1}$ L'arrêté du 20 juillet 2010 fixe une première tranche de neuf UHSA : au CH Gérard Marchand à Toulouse (Haute-Garonne), au Centre Psycho-thérapeutique de Nancy Laxou (Lorraine), au CH Paul Guiraud à Villejuif (Val-de-Marne), au CH Georges Daumezon à Fleury-Les-Aubray (Loiret), au CH Guillaume Régnier à Rennes (Ille-et-Vilaine), au CH Seclin à Seclin (Nord), au CH Édouard Toulouse à Marseille (Bouches-du-Rhône), et au CHS de Cadillac (Gironde).
} 
d'unités et représentants de l'Association des Secteurs de Psychiatrie en Milieu Pénitentiaire (David et al., 2012) dénoncent et critiquent vivement ces unités destinées au soin et qui placeraient la sécurité au premier plan, participant au virage punitif (Bérard, Chantraine, 2008 ; Mucchielli, 2008 ; Wacquant, 1996). En réponse, les pouvoirs publics et plusieurs psychiatres mettent en avant leur dimension sanitaire. Ils présentent ces unités comme un outil indispensable d'amélioration de l'accès aux soins en prison, qui a été un objet d'indignation publique récurrent au cours des dernières décennies, alimenté par la perception qu'un nombre croissant de personnes détenues ont des problèmes de santé mentale. Entre dénonciation du «sécuritaire » et justification par l'humanitaire, les débats soulèvent une incertitude sur les motifs de l'enfermement : est-il pénal, thérapeutique ou de protection du corps social?

Comment comprendre que la perspective d'une hybridation suscite une contestation si vive et donne lieu à des prises de position aussi polarisées, entre dénonciation du « sécuritaire » et justification par l'humanitaire ? Comment de telles unités ont-elles pu s'imposer de façon aussi massive dans ce contexte a priori hostile ? La forme d'hybridation qu'elles produisent est-elle effectivement inédite?

Pour répondre à ces questions, nous proposons de replacer la création des UHSA dans une analyse à plusieurs échelles du cadre juridique autorisant l'enfermement. Nous revenons d'abord sur la construction épistémique d'une régulation dichotomique de l'enfermement, entre hôpital psychiatrique et établissement pénitentiaire, et sur les différents projets d'hybridation de ces deux institutions élaborés depuis le XIX ${ }^{\mathrm{e}}$ siècle. Nous décrivons ensuite de manière socio-historique le développement des services psychiatriques en milieu pénitentiaire et les efforts qu'il suscite chez les psychiatres pour définir leur position vis-à-vis de l'enfermement. Dans un troisième temps, nous centrons l'analyse sur l'action publique de ces quinze dernières années qui conduit à l'ouverture des UHSA, et nous montrons que celles-ci s'inscrivent dans la continuité de cette histoire mais qu'elles en accentuent les tensions quant au motif de l'enfermement. Nous montrons enfin que la résolution de ces tensions, au travers de la production de règles encadrant le fonctionnement de ces unités, ouvre sur une interrogation concernant le statut de l'enfermement légal contemporain. Si ces différentes échelles s'appuient sur la complémentarité des travaux antérieurs des différents auteurs, elles se justifient surtout par l'intérêt d'introduire des interprétations pluridisciplinaires du cadre juridique autorisant l'enfermement. Ce cadre est composé de règles très diverses, de par leur processus d'élaboration, leur vocation normative comme leurs usages sociaux. La complémentarité de regards philosophique, juridique et sociologique permet d'éclairer comment les règles élaborées avec la création des UHSA répètent, actualisent et modifient les controverses autour du sens de l'enfermement.

Méthodologiquement, l'élaboration de l'article a conduit à mettre en commun les données empiriques recueillies par les auteurs au cours de leurs recherches respectives. Plusieurs corpus ont ainsi été constitués ${ }^{2}$. Le premier rassemble une littérature juridique et

\footnotetext{
${ }^{2}$ Les modalités détaillées de recueil des données empiriques sont présentées dans les travaux des auteurs (thèses en cours de Camille Lancelevée et Alexandre Litzer, Doron, 2011, Eyraud, Velpry, 2014b ; Protais, 2011 ; Saetta, 2012). Par ailleurs, si les enquêtes ethnographiques menées actuellement par Camille Lancelevée et Caroline Protais n'ont pas été directement utilisées, elles ont pu servir de point d'appui dans les analyses portant sur les usages sociaux des règles.
} 
psychiatrique sur la prise en charge des troubles mentaux en relation avec l'institution carcérale. Un second corpus est issu du recueil systématique des documents administratifs à leurs différents stades d'avancement, concernant le processus de création puis d'élaboration et de mise en fonctionnement des trois premières UHSA ouvertes. Enfin, un troisième corpus comprend une trentaine d'entretiens semi-directifs réalisés avec les acteurs ayant été impliqués dans la mise en place des UHSA, au sein des administrations sanitaires et pénitentiaires, et parmi les psychiatres. Le travail d'analyse des corpus a ensuite été mené collectivement au sein du Collectif Contrast ${ }^{3}$.

\section{L'asile ou la prison? Un régime dichotomique de l'enfermement et ses aménagements}

Au cours du $\mathrm{XIX}^{\mathrm{e}}$ siècle en France, un régime dichotomique de régulation ${ }^{4}$ de l'enfermement, soit en prison, soit à l'asile se met en place. Les difficultés qu'il pose dans plusieurs situations conduisent à des tentatives de transformation ou d'aménagement.

\subsection{Le régime dichotomique des régulations pénales et médico-sanitaires de l'enfermement}

$\mathrm{Au}$ lendemain de la période révolutionnaire, le régime qui s'instaure en matière d'enfermement - de l'élaboration de l'article 64 du Code Pénal à l'adoption de la loi de 1838 organisant le système asilaire - s'efforce d'établir une distinction ferme entre deux cadres légaux pour l'enfermement ${ }^{5}$ (Castel, 1976 ; Goldstein, 1997 ; Guignard, 2010), qualifié ici de « carcéral » et de «médico-administratif ».

L'enfermement carcéral, progressivement défini comme une peine, vise à sanctionner un délit ou un crime juridiquement constitués dans leurs différents éléments (légal, matériel et moral), et est soumis au contrôle de l'autorité judiciaire. L'élément matériel du délit ou du crime témoigne qu'il n'est pas question d'enfermer en prison quelqu'un au nom de bizarreries, d'immoralités, de troubles non qualifiables comme infraction, ni (théoriquement) de manière préventive, au nom d'un péril ou d'un danger qu'il ferait courir à la société. L'élément moral implique que la personne ait eu l'intention de nuire à travers son acte et puisse en être tenu responsable. Cristallisant ce principe, l'article 64 du Code pénal dispose qu' « il n'y a ni crime ni délit, lorsque le prévenu était en état de démence au temps de l'action, ou lorsqu'il a été contraint par une force à laquelle il n'a pas pu résister ».

D'autre part, l'enfermement médico-administratif, défini par la loi du 30 juin 1838, se fonde sur le constat d'un état d'aliénation mentale, ainsi que sur une demande des familles ou de l'autorité administrative et/ou sur la notion de « danger imminent». Cette procédure autorise donc à enfermer des personnes pour une durée indéterminée, au nom du danger qu'elles représentent et afin de leur apporter un soin, et ce sans contrôle judiciaire a priori mais à

\footnotetext{
${ }^{3}$ [http://contrastcollectif.wordpress.com/].

4 Nous utilisons ici ce terme dans le sens relativement souple d'une articulation originale de logiques institutionnelles, de groupes d'acteurs et de rationalités distinctes (judiciaire, psychiatrique, pénitentiaire...).

5 Une telle présentation est à visée heuristique. Il existe des dispositifs d'internements politiques et administratifs (prisons de haute police, dépôts de mendicité...) et de mécanismes permettant d'enfermer préventivement et postérieurement à la peine en prison. Voir Petit (1990).
} 
travers un filtre médical et/ou de la notoriété publique. Elle vise explicitement à éviter aux aliénés d'être retenus en prison, comme c'était souvent le cas, puisqu'elle prévoit dans son article 24 que «dans aucun cas, les aliénés ne pourront être ni conduits avec les condamnés ou les prévenus, ni déposés dans une prison ».

L'enfermement médico-administratif couple une vocation thérapeutique et protectrice de l'aliéné, qu'il s'agit de soigner à travers «l'isolement » et le «traitement moral » et de placer sous la tutelle de l'État; et une vocation de protection de la société, qu'il s'agit de protéger du péril potentiel causé par l'aliénation.

Dès sa mise en place, ce régime dichotomique soulève des difficultés dans les situations d'entre-deux, où l'enfermement pourrait relever aussi bien du carcéral que du médicoadministratif, ce qui conduit à rechercher des solutions intermédiaires, hybrides.

\subsection{Des situations d'entre-deux}

La première difficulté réside dans l'alternative entre responsabilité et irresponsabilité. Elle laisse échapper une zone trouble où les personnes sont dans des états qui, sans relever à proprement parler de la démence au moment de l'acte, sont susceptibles d'altérer leur volonté et leur capacité à se contrôler. Elle fait émerger une population de «l'entre-deux », qualifiée de «dégénérés », «déséquilibrés » ou «anormaux mentaux », au nom de laquelle les mouvements de défense sociale, à la fin du $\mathrm{XIX}^{\mathrm{e}}$ siècle, réclament le passage à un autre régime de pénalité, fondé sur la dangerosité d'une personne plutôt que sur la responsabilité d'un sujet vis-à-vis de son acte (Foucault, 1981; Tulkens, 1988; Doron, 2008 ; van de Kerchove, 2010).

Des solutions envisagent d'aménager la régulation dichotomique de l'enfermement; traitement médico-répressif, traitement [...] médico-socio-éducatif, art médico-pénitentiaire, loi psychiatrico-psychologico-juridique (Levasseur, 1959, 15), centres «socio-médicojudiciaires de rétention de sûreté $»^{6}$ (Pratt, 1998 ; Doron, 2011). Au-delà de la variété des dénominations, toutes introduisent les techniques préventives de transformation des personnes dans le cadre pénal, ouvrant ainsi à une hybridation entre les deux cadres de l'enfermement, parfois revendiquée (Pinatel, 1946). Elle va de pair avec une relative subordination du judiciaire aux savoirs criminologiques et psychiatriques. Dans plusieurs pays, des centres de défense sociale sont mis en place dans cette perspective ${ }^{7}$. En France, en dépit de très nombreuses tentatives ${ }^{8}$ au long du $\mathrm{XX}^{\mathrm{e}}$ siècle, on tend plutôt à aménager le régime dichotomique existant en y introduisant des dispositions inspirées de la défense sociale comme la personnalisation de la responsabilité (Dreyfus, 2010) ${ }^{9}$. À partir des années

\footnotetext{
${ }^{6}$ Loi n ${ }^{\circ 2008-174 ~ d u ~} 25$ février 2008, validée par le décret n ${ }^{\circ 2008-562 ~ d u ~ C o n s e i l ~ C o n s t i t u t i o n n e l . ~}$

${ }^{7}$ On peut distinguer, selon les pays, des systèmes dits «alternatifs » où les sujets sont enfermés dès qu'ils sont reconnus comme «anormaux » et les systèmes dits cumulatifs où l'emprisonnement est suivi du placement en « centre de sûreté » (Pratt, 1998 ; Lancelevée, 2014).

${ }^{8}$ Ces tentatives sont résumées, pour la période 1910-1959, in Doron (2011, 69-79).

${ }^{9}$ En témoigne la circulaire Chaumié du 12 décembre 1905, qui aménage l'article 64 du Code pénal en exigeant que l'expert détermine si, indépendamment de la démence au moment de l'acte, la personne n'est pas atteinte « [d']anomalies mentales ou psychiques de nature à atténuer dans une certaine mesure sa responsabilité »Ce principe remplacera l'article 64, lors de la révision du Code pénal en 1994, dans le nouvel article 122.1.
} 
1990, plusieurs rapports et propositions de loi visant à développer des dispositifs d'évaluation et de prévention de la récidive des délinquants réputés «dangereux » renforcent cette tendance. Ils se traduiront finalement en 2008 par la création de «centres de rétention de sûreté ${ }^{10}$, où l'enfermement est motivé par une peine et par une mesure de sûreté indéterminée indexée sur la dangerosité. L'ouverture concomitante des UHSA alimentera d'ailleurs la confusion sur le statut de l'enfermement dans ces dernières.

La seconde difficulté réside dans la prise en charge par la société des personnes ayant bénéficié d'une irresponsabilité pour des actes déviants qu'ils ont commis. Doit-on les enfermer à l'asile avec les autres aliénés ou bien créer pour eux des « sections spéciales », voire des «asiles spéciaux pour aliénés criminels »? Cette question reste en débat tout au long du XIX ${ }^{\mathrm{e}}$ siècle, avec une grande variété de solutions selon les pays et les localités ${ }^{11}$. Une solution qui dure dans sa forme administrative jusqu'à aujourd'hui émerge avec la création du «service de sûreté » de Villejuif, qui devient la première « unité pour malades difficiles » (UMD), dont l'objectif est de désengorger l'asile des patients présentant des comportements « criminels » ou très perturbateurs (Colin, 1912 ; Fau-Vincenti, 2013). Ces unités mêlent des malades ayant bénéficié d'une déclaration d'irresponsabilité avec des patients réputés particulièrement rétifs aux règles asilaires, au nom de leur commune dangerosité particulière (Velpry, à paraître). L'enfermement appliqué, particulièrement strict, relève toutefois des mêmes règles que le système asilaire classique. Après une certaine marginalisation de ces unités au cours d'une longue période marquée au contraire par une certaine ouverture des hôpitaux psychiatriques, elles sont fortement soutenues depuis quelques années, créant là encore une confusion avec les UHSA pour nombre d'acteurs (Eyraud, Velpry, 2014b).

Enfin, la troisième difficulté réside dans les réponses apportées aux personnes qui ne sont pas jugées aliénées au moment de l'acte mais qui s'avèrent présenter des troubles mentaux et avoir besoin de soins psychiatriques, en particulier en prison. Dès les années 1840, nombre de travaux font état d'une surreprésentation des malades mentaux en prison ${ }^{12}$, auxquels s'ajoutent progressivement des dénonciations du fait que de nombreux aliénés passent inaperçus au moment du jugement et parfois ensuite en prison même (Pactet, Colin, 1894 ; Régis, 1909).

Comment soigner ces personnes et dans quel cadre ? Faut-il, lorsque le sujet devient dément après sa condamnation, suspendre sa peine le temps que dure l'état de démence ? Préconisée

\footnotetext{
${ }^{10}$ Sur ces évolutions, et le rôle de l'argument du « soin » ou du «traitement » dans la légitimation ces mesures de sûreté, voir Danet (2009), Doron (2008, 2009, 2010) et Cartuyvels, Lancelevée et al. (2012).

11 Dès 1834, Ferrus recommandait pour les aliénés qui ont du penchant au meurtre ou au vol, dits aliénés criminels, la création d'une section spéciale (Ferrus, 1834). En 1844, la section de sûreté de Bicêtre réunira « les aliénés condamnés et les aliénés les plus dangereux » (voir Parchappe, 1853 ; Falret, 1869).

${ }^{12}$ Ces questions apparaissent souvent dans le vif débat sur les aliénations induites par le régime pénitentiaire luimême (la «folie pénitentiaire »), notamment dans les années 1835-1850, en lien avec la réforme des prisons et la question de l'isolement cellulaire. Voir les discussions par Moreau-Christophe $(1839,1844)$.
} 
par une première rédaction de l'article 64 (Guignard, 2010), cette option ne sera pas mise en œuvre ${ }^{13}$.

Faut-il transférer les détenus ayant besoin de soin dans les asiles d'aliénés ? Régulièrement pratiqué depuis le $\mathrm{XIX}^{\mathrm{e}}$ siècle, le transfert est longtemps réglé par la circulaire du 15 avril 1833, puis par l'article D398 du Code pénal. Si une réglementation particulière autorise ainsi l'enfermement médico-administratif de personnes qui relèvent déjà d'un régime d'enfermement pénitentiaire, le recours à cette solution reste cependant circonscrit par les motifs - le détenu doit présenter une agitation ou une dangerosité pour lui-même ou autrui en détention - et par la durée, qui doit être limitée ${ }^{14}$. Cet arrangement conserve donc un caractère exceptionnel.

Faut-il alors créer des quartiers spéciaux au sein des prisons pour accueillir ces détenus, des asiles-prisons (Constant, 1897) ? Des diverses expériences menées sur cette question très débattue à la fin du XIX ${ }^{\mathrm{e}}$ siècle, la plus connue est la Maison Centrale de Gaillon, où est créé en 1876 un premier quartier réservé aux «détenus aliénés ». Elle ne durera pas, mais attire l'attention sur la question du rôle des médecins aliénistes en milieu carcéral ${ }^{15}$, et de leur participation à la régulation pénitentiaire de l'enfermement. Le psychiatre doit-il se limiter à offrir des soins aux détenus qui en ont besoin ${ }^{16}$ ? Ou doit-il s'impliquer dans le processus pénal, comme le proposeront les tenants de la défense sociale, en participant à un dispositif d'évaluation de l'état mental et de la personnalité des détenus qui vise à mieux ajuster la peine?

La perspective de longue durée montre que les solutions désignées sous le terme de « défense sociale », qui reviennent sur le principe de dichotomie entre régulations pénale et médicoadministrative, ne seront pas véritablement adoptées en France. Les solutions effectivement mises en place pour les situations d'entre-deux aménagent et s'inscrivent dans un des régimes d'enfermement, celui de l'asile ou celui de la prison (Protais, 2011 ; Cartuyvels et al., 2012 ). Ce faisant, elles maintiennent une incertitude quant au motif, pénal ou thérapeutique, de l'enfermement. Cette incertitude est au cœur des difficultés que rencontrent les psychiatres dans le soin apporté aux détenus, comme nous allons le voir en examinant le développement de services psychiatriques dans les établissements pénitentiaires.

\footnotetext{
${ }^{13}$ La loi n 2014-896 du 15 août 2014 relative à l'individualisation des peines et renforçant l'efficacité des sanctions pénales crée la suspension de peine pour motifs psychiatriques. Voir Poncela (2014, 611).

${ }^{14}$ La circulaire de 1833 stipule ainsi que le transfert n'est fondé que si « le condamné atteint d'aliénation [est] cause de désordre », que sa maladie n'est pas incurable et qu'il n'est pas possible de l'isoler des autres détenus au sein de la prison (Parchappe, 1853).

${ }^{15}$ Pactet et Colin (1894) proposent par exemple une définition large : ils recommandent d'étendre le mandat de l'expertise, d'instaurer un examen systématique de l'état mental des prévenus comme des détenus et de créer des médecins-inspecteurs aliénistes dans les prisons.

${ }^{16}$ Les psychiatres sont présents en prison de façon continue depuis la fin du 19ème siècle mais en faible nombre. Des “annexes psychiatriques" ont ouvert dans les années 1930, d'abord à Loos-les-Lille, puis par le décret du 31 mars 1936, dans divers services de prisons parisiennes (La Santé, Fresnes...) (Renneville, 2011).
} 


\section{Des psychiatres à l'intérieur de la prison : se distancier de l'enfermement carcéral}

L'intervention de la psychiatrie en milieu carcéral est une solution «hybride » qui se développe fortement dans la seconde partie du $\mathrm{XX}^{\mathrm{e}}$ siècle. Afin de comprendre comment les UHSA s'imposeront comme solution institutionnelle dans les années 2000, nous apportons un éclairage socio-historique sur la psychiatrie pénitentiaire centrée sur la position des psychiatres vis-à-vis de l'enfermement des détenus.

\subsection{Après-guerre : la critique des enfermements}

Au lendemain de la Seconde Guerre mondiale, la réforme Amor entend développer l'offre de soins psychiatriques en prison. Les évolutions que connaissent les hôpitaux psychiatriques à partir de cette période sont un des facteurs qui conduisent à l'émergence d'une politique globale (Bellanger, 2014).

Avec la découverte de nouvelles pharmacopées et les orientations nouvelles des politiques de santé, les hôpitaux psychiatriques s'ouvrent, le nombre de lits baisse et le recours aux mesures légales d'enfermement diminue considérablement, ce qui transforme le rôle de l'hospitalisation dans les soins psychiatriques (Eyraud, Velpry, 2014a). Cette évolution conduit à un double phénomène : d'une part, des malades mentaux délinquants qui étaient dans le passé estimés irresponsables du fait de leurs troubles psychiatriques par les experts psychiatres et les magistrats sont responsabilisés. Ces malades passent dès lors de l'enfermement psychiatrique à l'enfermement carcéral. D'autre part, la gestion des troubles mentaux des détenus tend à se déplacer de l'hôpital vers la prison. Ces deux phénomènes s'enracinent dans une forte réticence du milieu hospitalier vis-à-vis de personnes qui présentent des troubles psychiatriques couplés à des problèmes de comportement à l'heure de l'humanisation et de l'ouverture des services (Protais 2011). Dès le début des années 1960, des psychiatres exerçant en prison soulignent cette double tendance, qu'ils attribuent à l'introduction des traitements pharmacologiques, à la fois faciles à administrer et diminuant fortement l'agitation des détenus ${ }^{17}$.

Alors que l'intervention des psychiatres en prison se développe, les liens entre enfermement psychiatrique et carcéral suscitent le soupçon, largement nourri par les théories critiques de la psychiatrie qui associent enfermement et contrôle social et fleurissent à cette période (Farges, $2013)^{18}$. Plusieurs associations se créent, relayant des mouvements de contestation menés par

\footnotetext{
17 Les médicaments ont permis, en moins de dix ans, de renverser la politique sanitaire en clinique criminologique. On s'est aperçu qu'au lieu d'envoyer le délinquant mental à l'hôpital psychiatrique - ce qui nécessitait le maintien d'une forteresse au sein de cet hôpital qui finit à peine d'abattre ses grilles et ses murs il était beaucoup plus simple de faire venir le psychiatre à la prison, avec son flacon de Largactil sous le bras. La dangerosité qui, autrefois, quand elle devenait paroxystique, constituait pour l'administrateur pénitentiaire un critère d'éviction, n'entraîne plus automatiquement le passage à l'hôpital psychiatrique (Gonin, 1966). Le nombre de détenus internés à l'hôpital psychiatrique du Vinatier passe de 22 à 8.

${ }^{18}$ Les paragraphes qui suivent s'appuient largement sur l'analyse d'Éric Farges dans sa thèse consacrée à la réforme des soins en milieu pénitentiaire.
} 
des détenus au sein d'établissements pénitentiaires ${ }^{19}$, pour dénoncer aussi bien l'enfermement carcéral que psychiatrique, suspectés de participer à la même vocation de contrôle social.

En réaction, les psychiatres marquent leur distance avec l'enfermement carcéral dans leurs prises de position publiques. Les instances de représentation professionnelle veillent à se distinguer d'un rôle de contrôle social incarné par les services pénitentiaires et à éviter la confusion des rôles (Ayme, 1997, 561). En 1974, le syndicat des psychiatres hospitaliers refuse ainsi de collaborer avec la sphère judiciaire pour imaginer une institution hybride de défense sociale. Dans une lettre adressée à la commission Pageaud, qui doit réviser l'article 64 du Code pénal et notamment concevoir des mesures combinant logiques psychiatrique et pénale pour les individus estimés partiellement responsables de leurs actes, il refuse catégoriquement la responsabilité de prendre en charge cette population :

«S'il existe un problème de délinquants anormaux mentaux, dont il est compréhensible qu'ils puissent avoir des difficultés à séjourner dans des services pénitentiaires courants, il appartient à l'administration pénitentiaire d'adapter ses services à sa propre clientèle » (SPH, 24 septembre 1974$)^{20}$.

Les psychiatres se distancient ainsi de l'enfermement de type carcéral dans un double mouvement. D'une part, ils reprennent la critique globale de l'enfermement et de son caractère répressif. D'autre part, ils réaffirment la dichotomie entre logiques pénale et psychiatrique dans la justification de l'enfermement. En ce qui concerne la politique de soins psychiatriques en prison, ce mouvement se traduira par l'émancipation de la psychiatrie visà-vis de l'autorité pénitentiaire et son inscription dans la politique de sectorisation psychiatrique.

\section{2.- Années 1970 : la défense des soins libres en prison}

À une période où les conditions de vie en prison font l'objet de réformes importantes dans le cadre d'une «politique d'humanisation » visant à faire de la peine de prison « une privation de liberté d'aller et de venir et rien d'autre » selon la formule du président de la République de l'époque, Valéry Giscard d'Estaing ${ }^{21}$, l'administration se montre très sensible au problème des “troubles psychiatriques". C'est un médecin psychiatre, Édith Rose, qui a rendu public les problèmes de santé au début des années 1970. L'affiliation des détenus à la Sécurité Sociale amorce une dynamique d'amélioration de l'accès aux soins somatiques en prison ${ }^{22}$. C'est encore un médecin psychiatre qui sensibilise l'administration pénitentiaire au problème des troubles psychiatriques : Nous sommes submergés de malades psychiatriques très agités qui

\footnotetext{
${ }^{19}$ Ces mouvements dénoncent tout particulièrement les conditions de vie en prison et défendent « les droits des détenus », en s'appuyant sur des textes internationaux, d'abord "ONU" de 1955, puis du conseil de l'Europe en 1973.

${ }^{20}$ La critique foucaldienne qui stigmatise fortement la collaboration justice/ psychiatrie dans ses cours sur les Anormaux tenus au Collège de France en 1974-75 pourrait influencer ce refus. Cette hypothèse mérite des travaux plus étoffés sur la réception des théories critiques de la psychiatrie chez les psychiatres français et notamment les représentants syndicaux de cette période. Voir cependant Protais (2011) et Farges (2013).

${ }^{21}$ Formule prononcée le 10 août 1974 après un déplacement à la prison de Lyon Saint-Paul.

${ }^{22}$ La loi du 29 janvier 1975, qui devient l'article L. 242-4 de Code de la Sécurité sociale, stipule ainsi que les détenus sont affiliés aux assurances maladie et maternité de la Sécurité sociale.
} 
n'ont plus rien à faire dans nos services et que l'on n'arrive pas à faire réintégrer au Centre psychiatrique [CMPR] de La Santé, faute de places $^{23}$.

Les discussions qui s'engagent alors à propos des soins psychiatriques portent non seulement sur l'offre de soin mais aussi sur l'autonomie de la psychiatrie carcérale. La circulaire de $1967^{24}$ avait transformé les six annexes psychiatriques toujours en fonctionnement en centres placés sous l'autorité conjointe du chef d'établissement et du directeur régional des services pénitentiaire. Le psychiatre n'y avait qu'une autorité médicale et le respect du secret médical était secondaire, puisque les médecins étaient tenus de donner les indications nécessaires à l'application du régime pénitentiaire du détenu.

Lorsque le groupe de travail Santé-Justice mis en place par la Chancellerie en 1974 propose d'intégrer ces centres au secteur psychiatrique (Hivert, 1983, in Farges, 2013), les réactions du syndicat des psychiatres hospitaliers sont dominées par la difficulté croissante à assumer l'enfermement, carcéral comme psychiatrique. Ils craignent en effet que ce rapprochement d'avec la psychiatrie pénitentiaire amalgame la psychiatrie à une activité de contrôle social, associée à l'asile et à la prison, alors même que les psychiatres des hôpitaux ont mis plus de 120 ans à sortir de l'asile (Fortineau, 1977, in Farges, 2013, 205). Si les représentants de la psychiatrie publique se rallient finalement au projet, c'est en partie dans la perspective qu'il allège les services de psychiatrie générale de leur mission d'enfermement, concernant les patients les plus agités, jugés indésirables (Farges, 2013).

L'administration pénitentiaire, l'administration des hôpitaux, et les représentants des psychiatres s'entendent ainsi finalement sur la circulaire interministérielle Santé-Justice du 28 mars 1977, qui émancipe officiellement le soin psychiatrique en prison de l'administration pénitentiaire en l'inscrivant dans l'organisation de la politique de sectorisation psychiatrique. Cette autonomisation est novatrice et influencera la réforme de 1994 concernant les soins somatiques. La mise en place en $1986^{25}$ d'au moins un Service Médico-Psychologique Régional (SMPR) par région pénitentiaire renforce cette dynamique. En $1988^{26}$, une circulaire rappelle que le code de déontologie - et donc le secret médical - s'applique en prison et réaffirme l'autonomie professionnelle des psychiatres vis-à-vis de l'administration pénitentiaire.

S'établit ainsi une répartition claire entre l'administration pénitentiaire, en charge de la garde, entendue comme détention des prisonniers, et les équipes psychiatriques qui interviennent pour soulager les détenus, mais qui ne participent pas à leur enfermement et au déroulé de la peine. Une telle répartition veut éviter le «double enfermement», pénal et thérapeutique. Cette doctrine ne cesse d'être réaffirmée, comme en atteste la recommandation de « ne jamais confondre contrainte de soins et contraintes liées à l'incarcération » d'un rapport sur la santé mentale et l'organisation des soins psychiatriques en milieu pénitentiaire (Gubler,

\footnotetext{
${ }^{23}$ Lettre du Médecin-chef Jacques Petit de l'Hôpital de Fresnes à Solange Troisier datée du 7/12/1973, in Farges (2013, 204).

${ }^{24}$ Circulaire du 30/09/1967 réglementant le fonctionnement des Centres médico-psychologiques régionaux (CMPR).

${ }^{25}$ Décret $n^{\circ}$ 86-602 du 14 mars 1986 relatif à la lutte contre les maladies mentales et à l'organisation de la sectorisation psychiatrique.

${ }^{26}$ Circulaire $n^{\circ} 1164$ du 5 décembre 1988 relative à l'organisation de la psychiatre en milieu pénitentiaire.
} 
Tcheriatchoukine, 1992) ${ }^{27}$. Cependant, l'accord ainsi négocié sur la répartition des rôles visà-vis des détenus ne se préoccupe que du soin délivré au sein de la prison. Il ne tient pas compte de la possibilité qu'ont les psychiatres d'hospitaliser les détenus dans un service de psychiatrie générale et ne cherche pas à en encadrer la pratique. Ainsi, la circulaire ne mentionne pas l'article D398 du Code pénal, qui permet l'hospitalisation sans consentement du détenu et son transfert à l'hôpital. Or, cette pratique perdure et constitue de fait une situation de «double-enfermement » qui constituera un nouveau point d'achoppement à la fin des années 1990.

\subsection{Années 1990 : les impasses de l'organisation des soins psychiatriques en prison}

L'hospitalisation d'un détenu à l'hôpital pour y recevoir des soins se pratique couramment, pour les soins somatiques comme psychiatriques. Il constitue un point d'achoppement récurrent entre l'administration de l'hôpital et l'administration pénitentiaire qui se cristallise autour de la responsabilité de la garde des détenus hors de la prison (pendant le transfert et à l'hôpital) et amène souvent à limiter l'accès aux soins des détenus ${ }^{28}$. À l'hôpital psychiatrique, le problème de la garde se pose de manière particulière, puisque c'est le personnel soignant étant supposé assumer cette mission ${ }^{29}$. Cette particularité du soin psychiatrique constitue une difficulté pour l'administration comme pour les équipes hospitalières, pourtant rarement discutée directement ${ }^{30}$.

D'abord, elle implique qu'un détenu ne peut faire l'objet d'une hospitalisation libre. Elle génère en outre des refus administratifs d'hospitalisation. Dans les années 1970, ces refus ont été nombreux, au motif de l'impossibilité d'assurer la garde dans les hôpitaux ; la direction de l'administration pénitentiaire déplore alors les effets négatifs de la sectorisation et de l'ouverture des hôpitaux (Bellanger, 2014). De plus, le développement des services médicopsychologiques régionaux n'a pas l'effet prévu de limiter «le transfert dans les hôpitaux spécialisés de malades » (Circulaire de 1977). Au contraire, dans les années 1990, le nombre d'hospitalisations de détenus croît rapidement ${ }^{31}$. Les observateurs incriminent de nombreux facteurs, parmi lesquels le mouvement de responsabilisation des malades mentaux par les experts psychiatres, la surpopulation carcérale, mais également l'augmentation des mesures de détention provisoire et la multiplication des procédures de jugement rapide, qui rendent difficile l'expertise psychiatrique (Bérard, Chantraine, 2008). La montée en puissance de l'intervention de psychiatres en prison, en intensifiant le repérage et la prise en charge des

\footnotetext{
${ }^{27}$ En pratique, cependant, le principe de non-intervention à la vie carcérale se confronte aux demandes pressantes de l'administration pénitentiaire, notamment en ce qui concerne un domaine partagé de compétence : la prévention du suicide. Voir la thèse en cours de Camille Lancelevée.

${ }^{28}$ Afin de répondre à cette difficulté pour les soins somatiques, sont créées en 1994 des unités hospitalières sécurisées (dites UHSI), au sein desquelles l'administration pénitentiaire assure la garde des détenus.

${ }^{29}$ L'article D 394 du code de procédure pénale qui prévoit que les détenus hospitalisés doivent être « gardés par un personnel de police ou de gendarmerie » précise que cette disposition ne s'applique pas aux détenus atteints de troubles mentaux.

${ }^{30}$ On l'a vu, les circulaires de 1977 et de 1986 concernant le soin psychiatrique en prison n'évoquent ni l'hospitalisation dans les établissements psychiatriques, ni la responsabilité de la garde pendant l'hospitalisation.

${ }^{31}$ L'administration pénitentiaire décompte une augmentation de 50\% de ces hospitalisations entre 1997 et 2000 , chiffres confirmés par la DREES, qui estime que le nombre d'hospitalisations de détenus est passé d'une centaine à la fin des années 1980 à 1500 en 2003 (Coldefy, 2007).
} 
troubles mentaux des détenus, participe sans doute aussi à développer sensiblement les demandes d'hospitalisation dans les services psychiatriques généraux.

Dans des services de psychiatrie générale qui sont de plus en plus souvent ouverts et au personnel réduit, les moyens mis en œuvre par les chefs de service consistent d'une part à réduire au maximum la durée de séjour des patients/détenus, et d'autre part à les placer en chambre d'isolement pour des motifs de sécurité. À la suite de nombreuses publications et rapports s'inquiétant de l'état de santé des détenus ${ }^{32}$, un rapport de l'Inspection Générale des Affaires Sociales centre l'attention sur les conditions d'hospitalisation psychiatrique des détenus et en synthétise les critiques. Y sont dénoncées «les durées d'hospitalisation anormalement courtes » et l'attitude soit laxiste, soit au contraire trop dure des psychiatres de secteur, relatant des cas où « le détenu se retrouve attaché en permanence à son lit, interdit de visite et de promenade, privé d'accès à la cantine et à la télévision, relégué dans une cellule d'isolement inadaptée » (IGAS, 2001).

Ces critiques sur le déroulement des hospitalisations nourrissent également le souci croissant pour les droits de la population détenue à bénéficier d'un accès au soin équivalent à celui de la population non incarcérée (Gonin, 1991). Parmi les réponses apportées à ces difficultés, les solutions qu'envisage le rapport de l'IGAS sont autant de propositions d'articulation entre les régulations carcérale et thérapeutique de l'enfermement : l'adoption pour la psychiatrie du modèle développé pour les soins somatiques, les UHSI, répartiraient strictement soin et garde entre administrations sanitaires et pénitentiaires ; l'ouverture d'unités fermées au sein des hôpitaux psychiatriques placerait en revanche le traitement du détenu mais également sa garde sous la responsabilité de l'autorité hospitalière. Dans ces années où l'attention se concentre sur l'hospitalisation des détenus, l'éventail des solutions envisagées concernant la responsabilité de l'enfermement est large. Pour autant, le débat ne semble pas avoir été encore complètement approprié par la communauté psychiatrique, puisque l'on ne trouve quasiment pas de publications de professionnels de la psychiatrie évoquant alors ce type d'unités. La mise en place des UHSA au début des années 2000 le rendra nécessaire.

\section{Participer à l'enfermement à reculons : des unités qui s'imposent sans convaincre}

Le 9 septembre 2002, la loi dite Perben prévoit que «Les personnes détenues, lorsqu'elles sont atteintes de troubles mentaux, sont hospitalisées dans des établissements de santé au sein d'unités spécialement aménagées ${ }^{33}$. En même temps que l'aboutissement du processus d'autonomisation de la psychiatrie au regard de l'administration pénitentiaire, cette disposition annonce une dynamique d'action publique qui conduit à un plan ambitieux de développement des UHSA, en même temps qu'il provoque l'embarras parmi de nombreux

\footnotetext{
${ }^{32}$ Outre les préoccupations que suscite leur augmentation, les hospitalisations des détenus alimentent les vives inquiétudes sur l'aggravation de la santé mentale des détenus (Vasseur, 2000), dont se font écho plusieurs études épidémiologiques et rapports publics au tournant des années 2000 (Voir Eyraud, Velpry, 2014b).

${ }^{33}$ C'est ainsi que, alors que les soins relèvent depuis 1997 du ministère de la Santé, c'est le ministère de la Justice qui introduit la création d'une nouvelle structure de soin. L'absence de concertation préalable avec le Ministère de la Santé entraînera quelques difficultés pour mettre en place le processus administratif.
} 
acteurs amenés à le mettre en œuvre, tout particulièrement les psychiatres intervenant en prison.

\subsection{L'arrivée discrète des UHSA}

À la suite du rapport de l'IGAS, un groupe de travail «santé/justice » s'est réuni avant le changement de majorité, en 2001 et 2002, dans lequel Catherine Paulet - alors chef de service du SMPR de Marseille - représente la psychiatrie en prison. Dans ses conclusions, qui ne seront publiées qu'en 2003, le groupe rejette toute solution tendant à la création d'établissements spécialisés pour les détenus atteints de troubles mentaux sous la tutelle des ministères de la Justice et de la Santé. Ces conclusions ne semblent pas avoir été discutées lors de la préparation de la loi, votée à l'automne 2002 par une nouvelle majorité politique préoccupée par les questions de sécurité et les moyens accordés à la justice ${ }^{34}$, qui insère l'article L 3214-1, et les «unités spécialement aménagées » dans le Code de la santé publique. La nouvelle structure ainsi créée vise à répondre aux problèmes posés par l'hospitalisation psychiatrique des détenus. Dans leur exposé des motifs ${ }^{35}$, les rédacteurs du texte répondent à quelques éléments mis en avant par les rapports précédents, en évoquant l'inadaptation des «services ouverts» dans les établissements psychiatriques, le souci de permettre une hospitalisation avec le consentement du patient et la complémentarité avec les UMD.

Une certaine confusion semble régner dans les services ministériels au sujet de l'origine et de la nature des UHSA. En effet, quand le directeur de la DHOS, Édouard Couty, annonce la phase opérationnelle de la création des UHSA, lors d'un colloque tenu en $2004^{36}$, il les présente comme une proposition du groupe de travail «santé/justice » qui a précédé la loi. Le directeur de l'administration pénitentiaire évoque, quant à lui, la création d'une nouvelle commission santé/justice, qui doit travailler sur «l'éventuelle création d'un hôpital prison ».

Les psychiatres intervenant en milieu pénitentiaire, de leur côté, se montrent ambivalents. Dans un premier temps, ils ont réagi froidement, craignant les effets pervers d'une solution qui développe les soins psychiatriques en prison. Ainsi, dans les premiers articles que consacrent Michel David - alors chef de service du SMPR de Guadeloupe - et Catherine Paulet au projet UHSA, le propos est circonspect face au risque de transférer en prison tous les malades qui nécessitent d'être enfermés: Alors, et si nous manquons de vigilance et de vertu, nous aurons tôt fait et avec bonne conscience (puisque des soins spécifiques de qualité existeront), d'accentuer la criminalisation des troubles mentaux pour évacuer la difficulté à soigner les patients les plus difficiles à soigner. Et nous aurons ainsi réussi une alternative à l'hospitalisation, la prison! (Paulet, 2004). Craignant une responsabilisation des malades

\footnotetext{
${ }^{34}$ Loi $\mathrm{n}^{\circ}$ 2002-1138 du 9 septembre 2002 d'orientation et de programmation pour la justice. Elle comporte des dispositions sur la justice de proximité, le droit pénal des mineurs, la simplification de la procédure pénale, la justice administration, la prise en charge des victimes, et l'amélioration et le fonctionnement des établissements pénitentiaires.

${ }^{35}$ Exposé des motifs [http://www.senat.fr/leg/pj101-362.html], consulté le 7 décembre 2014.

${ }^{36}$ Actes du colloque du 7 décembre 2004 «Dix ans après la loi : quelle évolution dans la prise en charge des personnes détenues ?» [http://www.vie-publique.fr/documents-vp/colloque_sante_prison.pdf].
} 
mentaux trop fréquente - par le recours à l'article 122-1 al. 2, notamment - et refusant la spécialisation des soins psychiatriques aux détenus, ils suggèrent plusieurs alternatives au projet (David, 2004). Malgré ces réticences, dès le colloque tenu en 2004 et précédemment cité, Catherine Paulet semble accepter l'idée d'une UHSA dont on peut espérer - si leur nombre et leurs moyens sont suffisants et si le travail d'articulation est le maître mot de leur projet médical - qu'elles amélioreront sensiblement les conditions d'hospitalisation qui sont actuellement déplorables (Actes du colloque du 7 décembre 2004, 96). Dans le même temps cependant, des critiques virulentes continuent à dénoncer les UHSA comme une fausse bonne idée (Dubret, 2008), qui chercherait en réalité à conjuguer soin psychiatrique et exclusion sociale, mesures de soin et mesures pénales.

Au sein de la communauté des psychiatres intervenant en prison, la création des UHSA génère ainsi d'abord une réaction ambivalente. La position critique dénonce implicitement ce qui est perçu comme l'instauration d'une logique de défense sociale masquée à l'UHSA. Elle réaffirme son attachement au partage entre les personnes « irresponsables » relevant du soin psychiatrique et les personnes « responsables » relevant de la prison, et donc au régime de régulation dichotomique de régulation pénale et psychiatrique évoqué dans la première partie. Une position plus pragmatique semble aussi se dessiner, qui souligne l'enjeu de la qualité des soins aux détenus. La haute administration, de son côté, semble encore peu volontariste.

\subsection{L’acceptation embarrassée des psychiatres «pénitentiaires »}

Une double pression, médiatique et juridique, va accélérer cette dynamique d'action publique. À la toute fin de l'année 2004, un double meurtre de soignants par un malade à Pau est très fortement médiatisé. À la suite de cet événement, le ministère de la Santé propose très vite un plan «santé mentale et psychiatrie 2005-2008 », où figurent des mesures concrètes de déploiement des UHSA et qui précise qu'elles feront l'objet d'un « volet à part entière de la politique de santé mentale à venir ».

En 2006, des pressions juridiques favorisent l'avancée du processus administratif. La Cour européenne des droits de l'homme condamne la France pour avoir maintenu en détention un détenu ayant des problèmes psychiatriques importants, malgré la disposition introduite dans le Code de la santé publique en $2002^{37}$. Quelques semaines plus tard, lorsque le Comité européen de prévention de la torture constate «l'état dramatique dans lequel se trouve la psychiatrie pénitentiaire en France » (CPT, 2007, 83), le gouvernement évoque dans sa réponse un « programme de construction de 17 unités d'hospitalisation spécialement aménagées (UHSA) » et prévoit l'ouverture d'une première unité à Lyon en $2009^{38}$.

Le programme connaît alors une accélération, à l'initiative conjointe des ministères de la Justice et de la Santé. De nombreux psychiatres participent aux réunions à l'invitation des ministères, l'argument pragmatique emportant in fine leur participation. Pierre Lamothe, qui dirige le SMPR à l'hôpital du Vinatier à Lyon et obtient que la première unité y soit ouverte,

\footnotetext{
${ }^{37}$ Arrêt Rivière contre France, 11 juillet 2006.

38 Réponse du Gouvernement de la République française aux demandes d'information urgentes du CPT concernant les paragraphes 200 et 201 du rapport relatif à sa visite en France du 27 septembre au 9 octobre 2006.
} 
expliquera ainsi en entretien que l'implication du ministère de la Justice et de l'administration pénitentiaire, si elle comporte un risque d'amalgame avec l'enfermement carcéral, offre une augmentation substantielle de moyens, inaccessible par le biais de la psychiatrie générale.

À partir de l'ouverture des négociations, l'enjeu, pour le personnel du ministère de la Santé comme pour les psychiatres est de tenir une position commune face à la Direction de l'Administration Pénitentiaire, dans un affrontement qui oppose les préoccupations de sécurité et thérapeutique. Les psychiatres adoptent donc un front commun autour de la défense du projet médical, tout en continuant à faire entendre des positions divergentes, où la crainte de la confusion entre enfermement carcéral et soin psychiatrique est vive :

Il faut être naïf pour croire que les pénitentiaires vont rester dehors les murs, et au milieu il y aura le personnel soignant... et les pénitentiaires n'interviendront qu'à l'appel de l'infirmier... Bien sûr que non! Non seulement ils ne vont pas intervenir qu'à l'appel de l'infirmier mais en plus ils vont mettre en place des systèmes de prévention. (...) Donc voilà c'est l'hôpital prison. Ce sont des prisons spéciales pour les malades mentaux.

Ces paroles sont prononcées par Évry Archer, qui déclare un peu après «les UHSA, c'est un scandale », mais sera appelé à concevoir l'UHSA de Lille. Elles montrent que réticence et embarras persistent, y compris parmi les psychiatres impliqués dans le processus.

Deux projets de soin écrits à la suite de ces réunions témoignent de cette position d'acceptation générale comme de ses modalités contrastées. La forme comme le ton des documents révèlent la différence de point de vue. Le projet écrit par Pierre Lamothe à Lyon est court mais dense, volontaire, sans embarras. À l'inverse, le pré-projet écrit par Évry Archer à Lille est plein de précaution et de réserve et se conclut sur le souci que l'UHSA ne devienne pas un hôpital-prison. Pour autant, les deux projets se rejoignent sur des arguments ou principes qui marquent la distance avec l'enfermement carcéral, et partant avec le soupçon sécuritaire. Chaque projet réaffirme non seulement l'autonomie de l'UHSA vis-à-vis de l'administration pénitentiaire dans la gestion des entrées et des sorties, mais aussi l'inscription de l'unité dans l'organisation des soins de psychiatrie générale. L'hospitalisation en UHSA vient compléter les soins ambulatoires apportés dans les services médicopénitentiaires et n'est aucunement liée à une dangerosité particulière des détenus, ce que les projets rappellent en soulignant la possibilité de transfert des détenus vers les unités pour malades difficiles, le cas échéant. Cette inscription est également appuyée sur le principe d'égal accès aux soins des détenus; les deux projets mentionnent la nécessité d'offrir la possibilité d'une hospitalisation consentie. Ils insistent enfin sur la séparation nette entre les domaines d'intervention du personnel sanitaire et ceux de l'administration pénitentiaire.

En somme, si les chefs de service intervenant en milieu pénitentiaire s'impliquent activement dans le projet de développement des unités spécialement aménagées, c'est avec la préoccupation de conserver une autonomie dans l'usage de l'hospitalisation psychiatrique vis-à-vis des prérogatives de l'enfermement carcéral. Ils portent ce souci dans les négociations avec le ministère de la Santé et le ministère de la Justice et l'inscrivent dans les projets de soin. Cette préoccupation d'éviter l'amalgame avec l'enfermement carcéral sera redoublée au moment de la mise en œuvre de ces unités au sein des hôpitaux psychiatriques, dans un contexte dominé par l'opposition entre dérive sécuritaire et mission soignante. 
Si à partir des années 2006 et 2007, la participation des psychiatres intervenant en prison au développement des UHSA semble acquise, ceux-ci doivent encore convaincre leurs établissements hospitaliers de rattachement. Certes, les directeurs d'hôpitaux sont sensibles à une politique de soutien financier fort à ces unités. Ils s'en saisissent comme une opportunité économique et foncière ${ }^{39}$, alors qu'ils sont par ailleurs en restriction financière. Cependant, cette dynamique contraste fortement avec les orientations générales des politiques de santé mentale du début des années 2000, qui restaient structurées par l'ouverture de l'hôpital vers la communauté.

La création des UHSA va ainsi être prise très vite dans l'opposition binaire, largement répandue parmi les professionnels de la santé mentale, entre «dérive sécuritaire » de la psychiatrie, dans laquelle les psychiatres se sentent enrôlés à leur corps défendant par les orientations gouvernementales, et logique de « soin », qui constituerait la « vraie » mission de la psychiatrie ${ }^{40}$. La tension entre ces deux pôles s'accroît dans les années où se construisent les UHSA, sous l'effet de différentes annonces du président de la République, le plus souvent suite à des faits divers. En août 2007, Nicolas Sarkozy crée ainsi l'émotion parmi les élus lyonnais en annonçant, après l'enlèvement d'un enfant, qu'un hôpital pour pédophiles va ouvrir à Lyon en 2009. Le psychiatre qui préside la Commission médicale d'établissement de l'hôpital du Vinatier dénonce la confusion introduite par le chef de l'État entre le projet en cours d'UHSA et la gestion de la dangerosité. Un peu plus d'un an plus tard, le discours dit d'Antony du 2 décembre 2008, qui annonce différentes mesures concernant la prise en charge des personnes présentant une dangerosité et la sécurité des hôpitaux ${ }^{41}$, symbolise, pour de nombreux psychiatres, l'assignation d'une mission «sécuritaire » à la psychiatrie et donne lieu à diverses mobilisations ${ }^{42}$.

Au sein des établissements porteurs d'un projet d'UHSA, l'annonce de ces mesures constitue une difficulté. À l'hôpital Paul-Guiraud de Villejuif, où existe déjà une UMD ancienne, l'UHSA encore en discussion est immédiatement associée à un développement sécuritaire. C'est, cette fois-ci, un sous-directeur qui le précise : Quand on a su que l'UHSA, c'était le projet Rachida Dati et le discours d'Antony de Nicolas Sarkozy, il a fallu beaucoup de méthode pour imposer l'UHSA au reste de l'hôpital. À l'hôpital du Vinatier à Lyon, le projet d'UHSA est parfois confondu avec le projet d'Unité pour malades difficiles en cours, y compris pour le psychiatre sollicité pour la diriger. À Lille, le choix du lieu d'implantation de l'UHSA suscite de vives résistances de la part d'une commune et de la direction d'un hôpital psychiatrique. De fait, l'ouverture de la première UHSA sera l'occasion de critiques

\footnotetext{
${ }^{39}$ La réorganisation de l'offre de soin en psychiatrie générale a laissé inoccupées de grandes emprises dans les anciens asiles psychiatriques.

40 Activement produite par les acteurs du champ, l'opposition entre «dérive sécuritaire » et «mission soignante » masque le fait que les partisans du «soin » ont joué un rôle considérable dans le développement du dispositif «sécuritaire » et sa légitimation, ainsi que d'importants effets de continuité, par exemple concernant l'héritage de la politique pénale « humaniste » dite de défense sociale nouvelle.

${ }^{41}$ Ce discours annonçait le doublement du nombre d'unités pour malades difficiles (UMD) et un plan de financement pour la sécurisation des hôpitaux psychiatriques.

${ }^{42}$ Un groupe de psychiatres et de soignants s'est ainsi réuni dans le «Collectif des 39 contre la nuit sécuritaire » [http://www.collectifpsychiatrie.fr/], consulté le 21/7/2012. Voir aussi la pétition : «Si c'est un homme, Appel contre les soins sécuritaires » [http://www.solidaires51.org/spip.php?article1246], consulté le 21/7/2012.
} 
particulièrement virulentes. Le jour de l'inauguration en mai 2010, différents collectifs, dont des syndicats de psychiatres et de personnels soignants, en résistance contre ce qu'ils appellent «l'idéologie sécuritaire », organisent une nuit blanche de protestation.

Dans ce contexte difficile, la mise en place de l'UHSA s'accompagne d'un processus de définition, tant administratif que clinique, qui vise à éviter l'amalgame avec le sécuritaire, sous sa forme d'enfermement carcéral comme de contrainte aux soins, et à accentuer la mission de réponse à la souffrance des détenus.

Ce souci de différenciation se traduit d'abord par des décisions organisationnelles. Lors des premières discussions, l'administration et les psychiatres impliqués envisageaient de regrouper les UHSA aves les soins en milieu pénitentiaire et les UMD dans un même pôle médico-administratif, afin de faciliter la gestion des ressources humaines et spatiales et de mutualiser formations et réflexions relatives aux protocoles de sécurité ou de gestion des risques. Dans le souci explicite de moins s'exposer aux critiques sécuritaires, les différents établissements accueillant des UMD et des UHSA rattachent ces deux types d'unités à des pôles distincts, suivant en cela la motion d'un syndicat de psychiatre ${ }^{43}$. Initialement favorable au rapprochement, le Dr Lamothe exprime finalement l'importance de cette distinction en termes d'affichage : faire apparaître un pôle de soin sous contrainte serait désastreux.

Du point de vue de la définition clinique des UHSA, dans les entretiens réalisés en 2012, les professionnels reprennent avec force deux arguments présents dans les projets de soin : le fait que les détenus hospitalisés ne sont pas dangereux et que la moitié d'entre eux sera hospitalisée librement, afin de se distinguer clairement de toute forme d'enfermement pour motifs thérapeutiques. Ils veillent également à dissocier très nettement le contexte d'enfermement carcéral où ils exercent, du soin qu'ils apportent au patient-détenu. L'administration pénitentiaire assure l'enfermement carcéral, sous la forme d'une sécurité de type périmétrique. Une fois passé ce «sas», les différents psychiatres chefs d'UHSA décrivent une situation de soin tout à fait ordinaire, libre de toute forme de contrainte et orientée vers le soulagement de la souffrance du patient.

Alors que les psychiatres intervenant en prison ont historiquement cherché à marquer leur distance d'avec l'enfermement carcéral, la création d'unités spécifiques pour les détenus réduit cette distance et les expose au risque de participer à aggraver encore le problème qu'ils dénoncent par ailleurs: la responsabilisation et l'incarcération croissantes de personnes présentant des troubles mentaux. Leur réticence face à une unité qui repose sur un « double enfermement», surnommée rapidement par la presse «hôpital-prison » prend également appui sur le risque d'une psychiatrie « sécuritaire ».

Si le contexte sécuritaire a très sûrement apporté les moyens pour réaliser les UHSA, la création de ces unités s'inscrit aussi dans un processus de résolution des tensions liées à la régulation dichotomique de l'enfermement. En conservant les deux régulations - carcérale et psychiatrique - de l'enfermement, la solution adoptée consiste plutôt à aménager la dichotomie dans une articulation originale. Les psychiatres impliqués soutiennent et renforcent cette option d'une hybridation a minima, au moment de l'ouverture au moins, en

\footnotetext{
${ }^{43}$ Il s'agit du syndicat des psychiatres des hôpitaux. Voir [http://penochet.fr/spip.php?article776].
} 
réaffirmant de façon parfois un peu caricaturale la distinction entre une fonction soignante porteuse d'un souci humanitaire mais dont le pouvoir d'enfermement serait occulté, et une fonction carcérale qui assurerait la sécurité. Cependant, l'ouverture des unités révèle la difficulté à maintenir cette distinction, ce qui se manifeste dans les règles de fonctionnement élaborées localement.

\section{La production locale de règles hybrides concernant l'enfermement}

La réticence des psychiatres à participer directement à une forme carcérale d'enfermement explique certainement la lenteur avec laquelle les règles relatives à l'organisation et au fonctionnement des UHSA ont été élaborées. De manière emblématique, la circulaire générale définissant ces unités ${ }^{44}$, annoncée dès le plan santé mentale en 2005 , ne sera publiée qu'un an après l'ouverture de la première UHSA. Tout en séparant explicitement les fonctions de sécurité et de soins, elle instaure a contrario de nombreuses dispositions spécifiques, qui ne relèvent ni complètement de l'administration pénitentiaire, ni de l'administration hospitalière. Dans cette dernière partie, nous nous appuyons sur l'analyse de cette circulaire et de règlements intérieurs locaux, ainsi que sur les relevés de décisions des «comités de pilotage UHSA » qui ont suivi l'ouverture de la première unité, à Lyon, pour montrer comment les discussions sur le fonctionnement des unités ont conduit à l'élaboration de règles nouvelles, marquant de fait une hybridation entre les règles de l'administration pénitentiaire et celles de l'hôpital. Ces règles locales hybrides s'élaborent relativement à trois dimensions de l'enfermement: celle du lieu de vie, des interventions qu'il permet sur les personnes, et enfin des entrées et sorties.

\subsection{L'hébergement et la vie quotidienne}

Le premier domaine est celui de l'hébergement et de la vie quotidienne dans lequel la question de la liberté d'aller et venir se pose de manière très concrète, aussi bien au niveau de l'architecture et des locaux, tout particulièrement des chambres, que de la manière d'y circuler (Litzler, 2013).

\section{Le statut des chambres/cellules}

La chambre de l'UHSA n'est pas tout à fait une chambre d'hôpital comme les autres ; elle a un statut hybride, du fait que le patient est aussi un détenu. Ainsi, alors que l'administration hospitalière autorise les patients hospitalisés en $\mathrm{SDRE}^{45}$ à rencontrer leur avocat dans la chambre du patient, suite à la réforme législative du 5 juillet 2011, cela ne sera pas le cas à l'UHSA. Le directeur représentant l'administration pénitentiaire s'y oppose, précisant que la personne hospitalisée n'est pas un patient, mais « un patient-détenu » (Comité de pilotage, 28 mars 2012). Au cours du comité de pilotage sont négociées un ensemble de règles mixtes concernant les chambres de l'UHSA. Les patients-détenus ont un accès à leur chambre qui

\footnotetext{
44 Circulaire interministérielle DGOS/R4/PMJ2/2011/105 du 18 mars 2011 relative à l'ouverture et au fonctionnement des unités hospitalières spécialement aménagées (UHSA).

${ }^{45}$ L'hospitalisation sur demande du représentant de l'Etat est définie par la loi du 5 juillet 2011, qui introduit également un contrôle judiciaire de la mesure et la possibilité pour le patient de rencontrer son avocat.
} 
reprend les règles de l'hôpital ${ }^{46}$, mais ils y disposent de certaines possibilités qui ont cours en cellule et sont issues des règles et usages de l'administration pénitentiaire.

Comme à l'hôpital psychiatrique, les patients-détenus peuvent être enfermés dans leur chambre à certains moments, non pas par du personnel pénitentiaire, mais par les soignants, qui ont les clés (Règlement intérieur, UHSA de Lille). D'une manière plus générale encore, les horaires d'ouverture des portes des chambres font l'objet d'une prescription médicale et relèvent de la prérogative soignante. Le projet de soin de Lille précise ainsi que "lorsque le patient est dans sa chambre, la fermeture de la porte ne sera effectuée que par le personnel soignant, sur prescription médicale ou à la demande du patient. Le patient ne peut être maintenu dans une chambre fermée que dans le respect strict des règlements hospitaliers et des recommandations de la conférence de consensus organisée par l'ANAES en juin 1998" (ibid.). En filigrane, ces dispositions révèlent la volonté de réaffirmer que l'équipe soignante, en gérant l'accès à la chambre, n'assure pas pour autant «la garde» des détenus mais applique des règles hospitalières.

Cependant, à l'UHSA les patients détenus peuvent fermer eux-mêmes la porte de leur chambre, y disposent de leurs effets personnels et y ont droit à la télévision individuelle. L'ensemble de ces caractéristiques, très inhabituelles à l'hôpital psychiatrique public, correspond aux règles et aux usages ayant cours en prison, où la cellule est un espace privé qui doit être protégé des empiètements de l'administration. Certains droits courants en prison sont malgré tout exclus; ainsi alors qu'il est possible de fumer et de se faire à manger dans sa cellule en prison, il est rappelé dans le règlement que ces deux choses sont interdites dans une chambre d'hôpital. L'hybridation des règles hospitalières et pénitentiaires a ainsi des effets contrastés sur la gestion de l'accès et de la disposition des chambres.

\section{Les déplacements/promenade}

Les règles concernant la circulation des patients-détenus à l'intérieur des unités révèlent également la construction d'une régulation hybride de l'enfermement. Dans les discussions, l'administration pénitentiaire fait état de sa préoccupation de respecter les règles de sécurité en cours en prison, tandis que l'administration sanitaire veut préserver à l'unité de soin son caractère ordinaire revendiqué dans la circulaire du 18 mars 2011. Dans les règles qui en résultent et sont formalisées dans le règlement intérieur, certaines missions de sécurité sont transférées du personnel pénitentiaire vers le personnel soignant.

Ainsi, la règle qui établit que «tout déplacement d'un détenu à l'intérieur de l'unité se fait accompagné par un membre de l'équipe soignante » est propre à l'UHSA et n'existe pas dans les unités d'hospitalisation ordinaire. Elle revient à faire porter par les soignants la préoccupation de l'administration pénitentiaire que les patients-détenus ne circulent pas seuls. Les soignants de l'UHSA doivent également respecter les consignes pénitentiaires interdisant que certaines personnes détenues se croisent - pour des motifs judiciaires ou proprement pénitentiaires.

\footnotetext{
${ }^{46}$ Il s'agit cependant d'une chambre d'hôpital telle qu'on les trouve dans les unités sécurisées. À l'UHSA de Nancy par exemple, tout le mobilier de la chambre est fixé au sol et les miroirs et verre sont feuilletés pour éviter de constituer des objets tranchants.
} 
De la même façon, la question des promenades revient à plusieurs reprises dans les discussions du comité de pilotage, car elle relève pour l'administration pénitentiaire d'un regroupement de personnes, qui fait l'objet de règles de sécurité spécifiques en prison. Au cours de ces discussions, "dans un but sécuritaire » (Comité de pilotage...), le nombre maximal de détenus-patients pouvant être dans la cour en même temps est négocié entre l'administration pénitentiaire et celle de l'hôpital.

Dans certains cas, les soignants eux-mêmes assurent la fonction de sécurité. À l'UHSA de Nancy, c'est une infirmière qui surveille les caméras installées dans l'unité de soin, là encore afin de maintenir le principe d'étanchéité entre l'espace thérapeutique et le périmètre pénitentiaire. Le personnel pénitentiaire n'a pas accès à ces caméras, sauf si le personnel soignant sonne l'alarme pour qu'ils interviennent.

\subsection{Intervenir sur les personnes : coopération entre sécurité et soin}

Si la circulaire de 2011 distingue très clairement les rôles et missions de chaque groupe professionnel, elle prévoit cependant également une coopération active entre équipes soignantes et équipes pénitentiaires, notamment pour le transport des patients-détenus, mais également pour la surveillance des personnes. Les soignants doivent ainsi s'assurer de la présence effective des patients, mais également signaler tout incident majeur ou disparition de matériel. Ils participent aussi de fait aux fouilles qui peuvent être intégrées à la démarche thérapeutique.

Certains impératifs de sécurité de l'administration pénitentiaire sont ainsi intégrés au sein du périmètre de soin et parfois même pris en charge par les soignants. À la demande de l'administration pénitentiaire, qui se réserve d'ailleurs le droit, «sans faire obstacle au bon déroulement des soins» de procéder aux fouilles de toutes les personnes détenues hospitalisées, des protocoles spéciaux sont prévus pour les DPS (détenus particulièrement surveillés). Ces documents définissent «les modalités retenues pour le soin et la sécurité durant la période d'hospitalisation du patient-détenu » et sont «cosignés par les Services Hospitaliers et Pénitentiaires » (Copil du 24 février 2011) ${ }^{47}$. Les personnels médicaux doivent notamment maintenir séparés certains patients si l'administration pénitentiaire le demande.

Cette coopération est un sujet particulièrement sensible lorsqu'elle concerne l'intervention du personnel pénitentiaire lors d'actes de soin, désignée sous le terme de " prêt de main forte ». En cas de difficulté avec un patient au cours d'une intervention thérapeutique, les soignants peuvent faire appel au personnel pénitentiaire «pour des raisons de sécurité » (Copil du 25 novembre 2010). En dernière extrémité, un soin technique, comme une injection de traitement, peut ainsi être imposé par le recours au personnel de la pénitentiaire. Cette hybridation des motifs de sécurité et de soin dans l'intervention directe sur la personne, dont le Comité européen pour la prévention de la torture soulignera qu'elle comporte un risque

\footnotetext{
${ }^{47}$ L'inscription de l'impératif de sécurité dans le travail soignant a pu avoir pour effet de créer une perception des patients de l'UHSA comme dangereux au sein de l'UHSA. Un psychiatre chef de service d'UHSA raconte que la formation des ambulanciers a été assurée par les agents ERIS - Équipes régionales d'intervention et de sécurité qui sont formées pour intervenir en cas de tensions dans les établissements pénitentiaires - "avec les mitraillettes, les gilets pare-balle qui ont effrayé tout le monde”. Et d'ajouter : “on n'a pas été assez soignant, les ambulanciers sont traumatisés ; ils pensent que le malade est un type dangereux
} 
pour le « respect de la dignité des patients » (CPT, 2012), doit voir ses modalités définies. À Lyon, la coopération sera formalisée par un protocole selon la difficulté des situations, qui limite l'intervention du personnel pénitentiaire aux alertes du niveau le plus élevé ${ }^{48}$.

Dans ces situations, la présence de l'administration pénitentiaire évite aux soignants de réaliser la dimension contraignante $\mathrm{du}$ soin. Ce faisant, une forme carcérale de l'enfermement, incarnée par les prêts de main forte assurés par l'administration pénitentiaire, participe à une dynamique thérapeutique.

\subsection{Entrer et sortir : l'UHSA et le système carcéral}

Le troisième domaine d'hybridation de l'enfermement réside dans les règles administratives d'entrée et de sortie dans l'UHSA et dans les établissements de provenance et de destination des patients détenus. Ces règles sont d'autant plus importantes qu'elles fixent la place de l'UHSA au sein du système carcéral et des différents dispositifs de psychiatrie pénitentiaire.

La question des entrées relève, d'un point de vue général, de la prérogative des psychiatres qui sont les seuls à pouvoir autoriser une hospitalisation dans l'unité. Mais les règles complexes des transferts, qui doivent être réalisés dans des conditions de sécurité particulière $^{49}$, les obligent à composer dans leur prérogative. Les psychiatres ont des usages différenciés de ces contraintes. Alors qu'à Lyon elles servent à justifier leur refus de réaliser des accueils de crise, à Lille elles permettent de revendiquer cette mission.

Les psychiatres décident aussi de la sortie d'hospitalisation. Cette prérogative est limitée par la levée d'écrou, qui oblige à une sortie immédiate du patient/détenu. Dans ce cas, la préparation de la sortie suppose une collaboration entre l'administration pénitentiaire, compétente pour la réinsertion des détenus ${ }^{50}$, et le service psychiatrique, à qui la circulaire du 18 mars 2011 délègue l'organisation de la fin de peine pour les patients-détenus nécessitant une hospitalisation.

La coopération entre l'administration pénitentiaire et l'équipe soignante implique que les soignants participent directement à des actes de sécurisation carcérale. Autrement dit, les deux corps professionnels régulent mutuellement leur exercice de la contrainte et de l'enfermement. Quelles sont les conséquences de cette élaboration d'une régulation hybride ? L'incorporation de la logique carcérale conduit parfois à des mesures plus restrictives des libertés qu'à l'hôpital, justifiées par l'impératif de sécurité pénitentiaire. Mais les usages du pénitentiaire apportent parfois une interprétation plus libérale du respect des droits des patients. Inversement, l'application de certaines règles propres à certains services hospitaliers sécurisés crée un environnement parfois plus contraignant par rapport à celui de la prison, qui fait dire aux patients-détenus, dans une formule souvent rapportée par les psychiatres, qu'ils « préfèrent la prison à l'hôpital ».

${ }^{48}$ À l'UHSA de Bron, le nombre d'interventions, initialement élevé, diminuera par la suite. À l'UHSA de Nancy, où environ 25 situations par an nécessitent l'intervention du personnel pénitentiaire, la psychiatre chef de service précise que les autres patients sont mis à l'écart pour qu'ils n'assistent pas à la scène.

${ }^{49}$ Les considérations administratives de gestion des heures supplémentaires des personnels participent aussi de la complexité des transferts.

${ }^{50}$ Compétences précisées l'article 2 de la loi pénitentiaire de novembre 2009 : la sécurité publique, la réinsertion et la prévention de la récidive ; la mission de réinsertion est menée par les SPIP (Bouagga, 2013) 


\section{Conclusion : une hybridation inévitable?}

Cette contribution trouve son point de départ dans les controverses qui ont accompagné le développement des UHSA en France. Nous avons postulé que ces controverses sont liées aux recompositions du sens de l'enfermement autorisé légalement dans le contexte social contemporain et qu'une analyse interdisciplinaire croisant la force normative des règles autorisant l'enfermement, le contexte de leur élaboration, et leurs usages sociaux, permettrait de les éclairer. Cette analyse a permis de dégager trois résultats.

Le premier rend compte du niveau de consensus qui rend inévitable la construction de ce type d'unités dans le contexte social contemporain. Les différents acteurs concernés considèrent que l'enfermement pénal ne doit pas conduire à une restriction d'autres droits. L'accès à toute l'offre de soins psychiatriques, et donc aux hospitalisations complètes, a constitué la matrice de l'action publique conduisant au développement des UHSA.

Le second rend compte du caractère ambigu des motifs qui conduisent à autoriser le recours à l'enfermement dans ces unités spécialisées et sur l'incertitude du niveau d'hybridation entre les motifs pénaux, thérapeutiques et préventifs d'enfermement. D'un côté, les règles générales qui les définissent séparent clairement le motif pénal de l'enfermement de l'activité thérapeutique et n'évoquent pas explicitement de dispositions préventives, telles que développées par les théories de «défense sociale ${ }^{51}$. D’un autre côté, la mise en œuvre de ces unités implique une production de règles locales hybrides qui mêlent fortement des motivations pénales, thérapeutiques, et mêmes préventives, laissant imaginer que des pratiques relevant de la défense sociale s'y développent ${ }^{52}$. Cette ambiguïté favorise les inquiétudes des acteurs attachés à une séparation claire des motifs pénaux et thérapeutiques de l'enfermement.

Le troisième résultat porte sur l'embarras des acteurs qui sont amenés à mettre en œuvre ces unités. L'analyse socio-historique des interventions psychiatriques en prison a montré que les psychiatres se sont progressivement autonomisés de l'administration pénitentiaire, afin d'éviter d'être considérés comme étant au service de l'enfermement carcéral et responsables d'un «double enfermement». Mais cette autonomisation n'a pas rendu possible le développement d'hospitalisation libre pour les détenus. Avec les UHSA, c'est le risque du double pouvoir d'enfermement contre lequel les psychiatres pénitentiaires s'étaient historiquement constitués qui a été réactivé.

L'identification des consensus, des ambiguïtés et des embarras des acteurs permet ainsi de comprendre pourquoi les UHSA, qui émergent de la tension entre le difficile accueil des

51 En cela, les UHSA constituent un aménagement et non pas une transformation de ce régime «dichotomique »: le juge judiciaire et le médecin psychiatre demeurent les garants que l'enfermement ne constitue pas un empiètement arbitraire aux libertés individuelles, ce régime trouvant sa rationalité dans l'indépendance de ces fonctions, et une certaine récusation de modèles « hybrides » donnant à certains acteurs le pouvoir d'enfermer aussi bien pour des raisons thérapeutiques que préventives.

52 L'analyse de l'usage des règles hybrides reste en cela à approfondir. Certaines observations conduisent à imaginer le développement notamment d'une «clinique du passage à l'acte », qui articulerait le travail thérapeutique à l'acte à l'origine de l'incarcération. Si cette question reste controversée parmi les soignants en UHSA, certains ont déjà mis en place des ateliers destinés à une intériorisation des interdits, une telle évolution pourrait facilement s'appuyer une conception «thérapeutique » de la responsabilisation notamment au nom d'une nécessité d'intérioriser la loi et l'interdit social, (Protais, 2011) que défendent certains psychiatres. 
détenus à l'hôpital psychiatrique et la revendication d'un accès au soin en santé mentale équivalent à celui offert par le système de soins de psychiatrie générale, sont un succès en termes d'action publique qu'aucun groupe d'acteur n'a véritablement porté et défendu et par rapport auquel les psychiatres restent ambivalents. Plus largement, ils font écho aux difficultés à actualiser le sens du régime dichotomique de régulation de l'enfermement.

Livia Velpry

livia.velpry@univ-paris8.fr

Benoît Eyraud

benoit.eyraud@ish-lyon.cnrs.fr

Centre Max Weber - CNRS

Institut des Sciences de l'Homme,

14, avenue Berthelot, 6

9007 Lyon

\section{Bibliographie}

AYME J., 1997, L'article 122-1 du Code pénal, ou comment ne fut pas résolu le paradoxe du fou criminel ?, in ALBERNHE T. (dir.), Criminologie et psychiatrie, Paris, Ellipses, 559-567 BELLANGER H., 2014, Politiques et pratiques de la psychiatrie en prison 1945-1986, Criminocorpus [En ligne], Savoirs, politiques et pratiques de l'exécution des peines en France au $\mathrm{XX}^{\mathrm{e}}$ siècle (25 et 26 mars 2014), Communications, mis en ligne le 01 décembre 2014, consulté le 26 décembre 2014 [DOI : 10.4000/criminocorpus.2730].

BÉRARD J., CHANTRAINE G., 2008, «80 000 détenus en 2017 », Réforme et dérive de l'institution pénitentiaire, Paris, Éditions Amsterdam/Vacarme.

BOUAGGA Y., 2013, Humaniser la peine ? Ethnographie du traitement pénal en maison d'arrêt, Thèse d'anthopologie soutenue à l'EHESS le 21 octobre 2013 sous la direction de Didier Fassin.

CARTUYVELS Y., LANCELEVÉE C., CLIQUENNOIS G., DUGUE F., BESSIN M., 2012, Ce que la dangerosité fait aux pratiques en prison: une comparaison BelgiqueFrance, Mission Droit et Justice, avril.

CASTEL R., 1976, L'ordre psychiatrique, Paris, Éd. de Minuit.

COLDEFY M. (dir.), 2007 La prise en charge de la santé mentale: recueil d'études statistiques, Paris, La Documentation française. 
COLIN H., 1912, Le quartier de sûreté de Villejuif, Annales médico-psychologiques, 2, 370391.

Comité européen pour la prévention de la torture et des peines ou traitements inhumains ou dégradants (CPT), 2007, $17^{\mathrm{e}}$ rapport général d'activités du CPT couvrant la période du $1^{\mathrm{er}}$ août 2006 au 31 juillet 2007.

Comité européen pour la prévention de la torture et des peines ou traitements inhumains ou dégradants (CPT), 2012, Rapport au Gouvernement de la République française relatif à la visite effectuée en France du 28 novembre au 10 décembre 2010.

CONSTANT C., 1897, Des aliénés criminels : nécessité d'une législation spéciale, Paris, Pedone.

DANET J., 2009, Les politiques sécuritaires à la lumière de la doctrine de la défense sociale nouvelle, Paris, Collège de France, 8 juin.

DAVID M., 2004, La place des unités d'hospitalisation spécialement aménagées (UHSA) dans le dispositif de soins aux détenus: leur application en Guadeloupe, L'information psychiatrique, 4, 80., 295-302

DAVID M., PAULET C., LAURENCIN. G., 2012, Psychiatrie en milieu pénitentiaire : la loi de 1994 pourrait-elle être remise en cause par l'essor de la préoccupation sécuritaire et de l'évaluation de la dangerosité ?, L'information psychiatrique, 88, 8, 605-15.

DORON C.-O., 2010, La volonté de soigner : d'un singulier désir de soin dans les politiques pénales, in LEFEVE C., WORMS F. (dir.), La philosophie du soin, Paris, PUF, 283-300.

DORON C.-O., 2009, Une volonté infinie de sécurité : vers une rupture générale dans les politiques pénales?, in CHEVALLIER P., GREACEN T. (dir.), Folie et justice : relire Foucault, Paris, Érès, 179-203.

DORON C.-O., 2008, La rétention de sûreté : vers un nouveau type de positivisme juridique ? », L'information psychiatrique, 6, 1, 533-541.

DORON C.-O., 2011, Races et dégénérescence. L'émergence des savoirs sur l'homme anormal, thèse de doctorat, Université Paris Diderot.

DREYFUS B., 2010, Regard contemporain sur la défense sociale nouvelle de Marc Ancel, Paris, L'Harmattan.

DUBRET G. 2008, UHSA : un formidable effort dans la mauvaise direction, L'information psychiatrique, 84, 543-550.

ESQUIROL, 1833, Questions médico-légales sur l'isolement des aliénés, Annales d'hygiène publique et de médecine légale, 9, 131-191.

EYRAUD B., VELPRY L., 2014a, De la critique de l'asile à la gestion de l'offre en santé mentale : une désinstitutionnalisation à la française de la psychiatrie ?, Revue Française d'Administration Publique, 149, septembre, 207-222.

EYRAUD B., VELPRY L., 2014b, Redéfinir la place de l'enfermement dans la pratique psychiatrique. L'exemple des unités sécurisées, in BUJON T., DOURLENS C., LE NAOUR G. (dir.), Aux frontières de la médecine, Paris, Édition des archives contemporaines, 165-180.

FALRET J., 1869, Des aliénés dangereux et des asiles spéciaux pour les aliénés dits criminels : discours prononcés à la Société médico-psychologique, le 27 juillet et le 16 novembre 1868, Paris, Impr. de E. Donnaud. 
FARGES E., 2013, Réformer l'organisation des soins dans les prisons françaises: les tentatives de spécialisation de la "médecine pénitentiaire »(1970-1994), Thèse de doctorat en Science Politique sous la direction de Gilles POLLET, 28 juin, Université Lumière Lyon 2.

FATOME T., VERNEREY M., LALANDE F., VALDES BOULOUQUE M., FROMENT B., 2001, L'organisation des soins aux détenus. Rapport d'évaluation, IGAS/ISGJ.

FAU-VINCENTI V., 2013, Vers les UMD : questionnements, tâtonnements et mise en œuvre, Gaillon (1876) et Villejuif (1910) sous l'égide du Dr Henri Colin, in GUIGNARD L., GUILLEMAIN H., TISON S., Expérience de la folie, criminels, soldats, patients en psychiatrie $\left(19^{e}-20^{e}\right)$, Rennes, Presses universitaires de Rennes, 69-80.

FAZEL S., DANESH J., 2002, Serious mental disorder in 23000 prisoners: a systematic review of 62 surveys, Lancet, 359, 545-550.

FERNANDEZ F. LÉZÉ S., 2011 Finding the moral heart of carceral treatment. Mental health care in a French prison, Social science and Medicine, 72, 9, 1563-1569.

FERRUS G., 1834, Des aliénés, Paris, Huzard.

FORTINEAU J., 1977, Les psychiatres des hôpitaux en prison ?, Bulletin des psychiatres des hôpitaux, 3.

FOUCAULT M., 1981, L'évolution de la notion d'individu dangereux, Déviance et Société, 5, 4, 403-422.

GASPARIN A., 1837, Rapport au Roi sur les prisons départementales, Paris, Imprimerie Royale.

GOLDSTEIN J., 1997, Consoler et classifier, Paris, Les Empêcheurs de penser en rond.

GONIN D., 1966, Une expérience de douze années à l'annexe psychiatrique de la Maison d'arrêt de Lyon, L'information psychiatrique, 9, 885-890.

GONIN D., 1991, La santé incarcérée : médecine et conditions de vie en détention, Paris, .L'Archipel

GUBLER C., TCHERIATCHOUKINE J., 1992, Rapport de synthèse sur les infirmeries des établissements pénitentiaires, Paris, IGAS, 1992/01.

GUIGNARD L., 2010, Juger la folie. La folie criminelle devant les Assises au XIX siècle, Paris, PUF.

HIVERT P., 1983, Le C.M.P.R., aspects historiques L'Information psychiatrique, 59, 2, 153 159.

LAMB H.R., WEINBERGER L.E., 1998, Persons with severe mental illness in jails and prisons: a review, Psychiatric Services, 49, 483-492.

LANCELEVÉE C., 2014, Soigner ou punir? Dilemmes européens, La Lettre du Psychiatre, décembre, 152-155.

LEVASSEUR G. (dir.), 1959, Les délinquants anormaux mentaux, Paris, Cujas.

LITZLER A., 2013, Soigner et surveiller en unités d'hospitalisation spécialement aménagées (UHSA), Archives de politique criminelle, 1, 35, 277-296.

MOREAU-CHRISTOPHE L., 1839, De la mortalité et de la folie dans les prisons, Paris, Renouard. 
MOREAU-CHRISTOPHE, L., 1844, Défense du projet de loi sur les prisons, Paris, Bureau de la revue pénitentiaire.

MUCCHIELLI L. (dir.), 2008, La frénésie sécuritaire. Retour à l'ordre et nouveau contrôle social, Paris, La Découverte.

PACTET F., COLIN H., 1894, Les aliénés dans les prisons (aliénés méconnus et condamnés), Paris, Masson.

PARCHAPPE M., 1853, Des principes à suivre dans la fondation et la construction des asiles d'aliénés, Paris, Masson.

PAULET C., 2004, Bilan et perspectives de l'organisation des soins psychiatriques aux personnes détenues, en particulier de l'hospitalisation, L'Information Psychiatrique, 80, 307312.

PETIT J.-G., 1990, Ces peines obscures. La prison pénale en France (1789-1870), Paris, Fayard.

PINATEL J., 1946, Chronique pénitentiaire, Revue de science criminelle et droit pénal comparé, 1, 142-143.

PONCELA P., 2014, Les peines extensibles de la loi du 15 août 2014, Revue de science criminelle et droit pénal comparé, 3, .611-622.

PRATT J., 1998, Governing the dangerous, Sydney, Federation Press.

PROTAIS C., 2011, Sous l'emprise de la folie. La restriction du champ de l'irresponsabilité psychiatrique en France (1950-2007), Thèse en sociologie dirigée par Nicolas Dodier, soutenue à l'EHESS le 12 décembre.

REGIS E., 1909, Précis de psychiatrie, Paris, Doin.

RENNEVILLE M., 2011, L'affaire Joseph Vacher : la fin d'un «brevet d'impunité » pour les criminels ?, Droit et cultures [En ligne], 60|2010-2, p. 129-142, mis en ligne le 17 mars.

SAETTA S., 2012, L'expertise psychiatrique dans les affaires criminelles : entre humanisme répressif et défense sociale de type managérial, Les cahiers de la justice, 3, 105-123.

TULKENS F., 1988, Généalogie de la défense sociale en Belgique (1880-1914), Bruxelles, Story-Scientia.

van DE KERCHOVE M., 1986, Discours juridique et discours psychiatrique. Aux sources de la loi de défense sociale, Droit et Société, 3, 279-302.

van DE KERCHOVE M., 2010, Les avatars de la loi belge de défense sociale, Déviance et Société, 4, 485-502.

VASSEUR V., 2000, Médecin-chef à la prison de la Santé, Paris, Le Cherche-Midi.

VELPRY L., (à paraître), Moderniser l'enfermement en psychiatrie. Le cas des unités pour maladies difficiles, Sociétés contemporaines.

WACQUANT L., 1996, De l'État charitable à l'État pénal : notes sur le traitement politique de la misère en Amérique, Regards sociologiques, 11, 30-38.

\section{Abstract}

In 2010, the first unit designed to hospitalize prisoners, called UHSA, was both criticized as security-driven and praised as promoting equal access to care. To understand how UHSAs 
developed in this contentious environment, we consider the rules authorizing confinement through an interdisciplinary perspective, integrating these rules' normative power, the context in which they were developed as well as their social use. Drawing on legal and administrative literature and on interviews, we show that the historical dichotomy in the regulation of confinement and the growth of mental health care in prison led to design rules for the UHSA that contribute to transform the meaning of confinement. 